\title{
FIXED ENERGY INVERSE PROBLEM FOR EXPONENTIALLY DECREASING POTENTIALS *
}

\author{
GUNTHER UHLMANN ${ }^{\dagger}$ AND ANDRÁS VASY $\ddagger$
}

1. Introduction. In this paper we show that in two-body scattering the scattering matrix at a fixed energy determines real-valued exponentially decreasing potentials. This result has been proved by Novikov previously [3], see also [2], using a $\bar{\partial}$-equation. We present a different method, which combines a density argument and real analyticity in part of the complex momentum. The latter has been noted in [2]; here we give a short proof using contour deformations, similarly to [1, Section 1.5]. We thus prove:

TheOREM 1.1. Suppose that $n \geq 3, V, V^{\prime} \in e^{-\gamma_{0}|w|} L^{\infty}\left(\mathbb{R}_{w}^{n} ; \mathbb{R}\right)$ for some $\gamma_{0}>0$, and $\lambda>0$. If $S_{+}(\lambda)=S_{+}^{\prime}(\lambda)$, then $V=V^{\prime}$. Here $S_{+}(\lambda)$, resp. $S_{+}^{\prime}(\lambda)$ are the scattering matrices of $H=\Delta+V$ and $H^{\prime}=\Delta+V^{\prime}$ at energy $\lambda$.

Theorem 1.1 for compactly supported potentials follows from an analogous result in [4] for the corresponding Dirichlet-to-Neumann map. See [5, Section 12], and the references given in these papers for a review of the relation between the Dirichlet-toNeumann map and the fixed energy problem.

The general method follows [4], as discussed in [1]. We thus recall the construction of complex exponential solutions $u_{\rho}, \rho \in \mathbb{C}^{n}$ of $(H-\lambda) u_{\rho}=0$, where $u_{\rho}(w)=$ $e^{i \rho \cdot w}\left(1+v_{\rho}(w)\right), \rho \cdot \rho=\lambda$, and $v_{\rho} \rightarrow 0$ in an appropriate sense as $\rho \rightarrow \infty$. These solutions exist for $\rho$ outside an 'exceptional set' which is discrete in $z$. We also show that if we write $\rho=z \nu+\rho_{\perp}, \nu \in \mathbb{S}^{n-1}, \rho_{\perp} \in \mathbb{R}^{n}$ perpendicular to $\nu$, and $z \in \mathbb{C} \backslash \mathbb{R}$, then for fixed $\nu, u_{\rho}$ is analytic in $z$ and real analytic in $\rho_{\perp}$, hence extends to be analytic in a neighborhood of $\mathbb{R}^{n-1} \backslash\{0\}$ in $\mathbb{C}_{\rho_{\perp}}^{n-1}$. The exceptional set is then given by the zeros of an analytic function of $z$ and $\rho_{\perp}$. We caution the reader that the extension of $u_{\rho}$ to complex $\rho_{\perp}$ does not agree with $u_{z \nu+\rho_{\perp}}$ where $\rho_{\perp}$ is allowed to be complex; indeed $v_{\rho}$ will merely lie in $e^{\gamma|w|} L^{2}\left(\mathbb{R}^{n}\right)$ for some $\gamma>0$.

We use this in the inverse problem as follows. Let $u_{\rho}, u_{\rho^{\prime}}^{\prime}$ be exponential eigenfunctions of $H$, resp. $H^{\prime}$, as above. Now consider the pairing

$$
\int_{\mathbb{R}^{n}} u_{\rho}\left(V-V^{\prime}\right) u_{\rho^{\prime}}^{\prime}
$$

where $\rho=z \nu+\rho_{\perp}, \rho^{\prime}=z^{\prime} \nu+\rho_{\perp}^{\prime}$, and $\nu$ is fixed. If $u_{\rho}, u_{\rho}^{\prime}$ are replaced by tempered distributional eigenfunctions of $H$ and $H^{\prime}$, then a standard argument shows that $S_{+}(\lambda)=S_{+}^{\prime}(\lambda)$ implies that the corresponding pairing vanishes. We employ a density argument to deduce that the pairing also vanishes for the complex exponentials provided that $\left|\operatorname{Im} z+\operatorname{Im} z^{\prime}\right|$ is small and $\rho \cdot \rho=\lambda=\rho^{\prime} \cdot \rho^{\prime}$. We then let $\rho, \rho^{\prime} \rightarrow \infty$. By analyticity, the pairing still vanishes. On the other hand, $v_{\rho}, v_{\rho^{\prime}}^{\prime} \rightarrow 0$, so for $\zeta=\rho-\rho^{\prime} \in \mathbb{R}^{n}$ we deduce that $\int_{\mathbb{R}^{n}} e^{i \zeta \cdot w}\left(V-V^{\prime}\right)=0$, i.e. the Fourier transform

\footnotetext{
*Received January 11, 2002; accepted for publication April 15, 2002.

$\dagger$ Department of Mathematics, University of Washington, Seattle, WA (gunther@math.washington.edu). G. U. is partially supported by NSF grant \#DMS-00-70488 and a John Simon Guggenheim fellowship.

${ }^{\ddagger}$ Department of Mathematics, Massachusetts Institute of Technology, Cambridge MA 02139, USA (andras@math.mit.edu). A. V. is partially supported by NSF grant \#DMS-99-70607. Both authors are grateful for the hospitality of the Mathematical Sciences Research Institute in Berkeley, CA.
} 
of $V-V^{\prime}$, hence $V-V^{\prime}$, vanish. In fact, this step will be slightly more complicated, because the density argument imposes restrictions on $\zeta$, and we first deduce vanishing of the Fourier transform of $V-V^{\prime}$ in a spherical shell of finite 'thickness', and then use the exponential decay of $V-V^{\prime}$ to conclude that it is in fact identically zero.

The authors are grateful to Maciej Zworski for his generous encouragement.

2. Exponential eigenfunctions. In this section we recall the construction of exponential solutions of $(H-\lambda) u=0$ from [4]. First, for $\rho \in \mathbb{C}^{n}$, let

$$
u_{\rho}^{0}(w)=e^{i \rho \cdot w} .
$$

Thus, $u_{\rho}^{0}$ is an 'exponential eigenfunction' of $\Delta$, namely

$$
(\Delta-\lambda) u_{\rho}^{0}=0, \quad \rho \cdot \rho=\lambda .
$$

We assume everywhere that $n \geq 3$.

For the Hamiltonian $H$, we then seek exponential solutions $u$ of the form

$$
u=u_{\rho}=e^{i \rho \cdot w}\left(1+v_{\rho}\right), \rho \cdot \rho=\lambda, \rho \in \mathbb{C}^{n},
$$

where $v_{\rho}$ is considered a perturbation. In fact, we will have $v_{\rho} \in L_{r}^{2}\left(\mathbb{R}^{n}\right)$ for all $r<0$ (when $v_{\rho}$ exists). Here $L_{p}^{2}=L_{p}^{2}\left(\mathbb{R}^{n}\right)$ denotes the $L^{2}\left(\mathbb{R}^{n},\langle w\rangle^{2 p} d w\right),\langle w\rangle^{s}=$ $\left(1+|w|^{2}\right)^{s / 2}$. Substituting $u$ into $(H-\lambda) u=0$, we obtain

$$
\left(\Delta+2 \rho \cdot D_{w}+V\right) v_{\rho}=-V .
$$

The construction given below works under power-decay assumptions on $V$, but we state it for exponentially decaying $V$, since $v_{\rho}$ is real analytic in the appropriate components of $\rho$ only in that case. So we assume that

$$
V \in e^{-\gamma_{0}|w|} L^{\infty}\left(\mathbb{R}^{n}\right), \gamma_{0}>0
$$

Thus, we need to construct a right inverse $G(\rho)$ to

$$
P(\rho)=\Delta+2 \rho \cdot D_{w}+V
$$

that can be applied to rapidly decreasing functions. Once this is done,

$$
u_{\rho}=e^{i \rho \cdot w}(1-G(\rho) V)
$$

is the solution to the original problem. Below we write

$$
P_{0}(\rho)=\Delta+2 \rho \cdot D_{w} .
$$

Since a right inverse $G_{0}(\rho)$ of $P_{0}(\rho)$ can be constructed explicitly, perturbation theory will give the existence of $G(\rho)$.

Namely, let

$$
G_{0}(\rho)=\mathcal{F}^{-1}\left(|\xi|^{2}+2 \rho \cdot \xi\right)^{-1} \mathcal{F}
$$

so $P_{0}(\rho) G_{0}(\rho)=$ Id e.g. on Schwartz functions. Thus, on the Fourier transform side $G_{0}(\rho)$ acts via multiplication by $\left(|\xi|^{2}+2 \rho \cdot \xi\right)^{-1}$ which is in $L^{1}\left(\mathbb{R}^{n}\right)$. It is convenient to represent $\rho$ as

$$
\rho=z \nu+\rho_{\perp}, \rho_{\perp} \in \mathbb{R}^{n}, \nu \in \mathbb{S}^{n-1}, z \in \mathbb{C}, \rho_{\perp} \cdot \nu=0 .
$$


We often identify $\operatorname{span}\{\nu\}^{\perp}$ with $\mathbb{R}^{n-1}$. For $z \notin \mathbb{R}$, this distribution is conormal to

$$
\begin{aligned}
S(\rho) & =\left\{\xi \in \mathbb{R}^{n}:|\xi|^{2}+2 \operatorname{Re} \rho \cdot \xi=0, \operatorname{Im} \rho \cdot \xi=0\right\} \\
& =\left\{\xi \in \mathbb{R}^{n}:\left(\xi+\rho_{\perp}\right)^{2}=\rho_{\perp}^{2}, \nu \cdot \xi=0\right\} .
\end{aligned}
$$

Note that $S(\rho)$ actually depends only on $\rho_{\perp}$ and $\nu$, not on $z$.

Below we write $e^{\gamma\langle w\rangle} L^{2}\left(\mathbb{R}^{n}\right)=L^{2}\left(\mathbb{R}^{n} ; e^{-2 \gamma\langle w\rangle} d w\right)$, and if $m$ is an integer,

$$
e^{\gamma\langle w\rangle} H^{m}\left(\mathbb{R}^{n}\right)=\left\{u \in e^{\gamma\langle w\rangle} L^{2}\left(\mathbb{R}^{n}\right): D^{\alpha} u \in e^{\gamma\langle w\rangle} L^{2}\left(\mathbb{R}^{n}\right),|\alpha| \leq m\right\} .
$$

The latter is equivalent to $e^{-\gamma\langle w\rangle} u \in H^{m}\left(\mathbb{R}^{n}\right)$, hence the notation.

We first recall:

Proposition 2.1. [4, Proposition 3.1], [6, Theorem 1.1] $G_{0}(\rho): L_{p}^{2} \rightarrow L_{r}^{2}$ is bounded for $p>0, r<0, r<p-1$. Moreover, the norm of $G_{0}(\rho)$ as a bounded operator between these spaces goes to 0 as $|\rho| \rightarrow \infty$.

Our central result is the following proposition.

Proposition 2.2. Suppose that $\gamma>0$ and fix $\nu \in \mathbb{S}^{n-1}$. Then there exists a neighborhood $U$ of $\mathbb{R}^{n-1} \backslash\{0\}$ in $\mathbb{C}^{n-1}$ and an operator

$$
\mathcal{G}_{0}\left(z, \rho_{\perp}\right): e^{-\gamma\langle w\rangle} L^{2}\left(\mathbb{R}^{n}\right) \rightarrow e^{\gamma\langle w\rangle} H^{2}\left(\mathbb{R}^{n}\right)
$$

defined on $(\mathbb{C} \backslash \mathbb{R}) \times U$ such that $\mathcal{G}_{0}$ is analytic on $(\mathbb{C} \backslash \mathbb{R}) \times U$ as a bounded operator between these spaces, and its restriction to $(\mathbb{C} \backslash \mathbb{R}) \times\left(\mathbb{R}^{n-1} \backslash\{0\}\right)$ is $G_{0}(\rho), \rho=z \nu+\rho_{\perp}$. Thus, for $z \in \mathbb{C} \backslash \mathbb{R}, \rho_{\perp} \in \mathbb{R}^{n-1} \backslash\{0\}$, the operator $G_{0}(\rho): e^{-\gamma|w|} L^{2} \rightarrow e^{\gamma|w|} L^{2}$ is complex-analytic in $z$, real analytic in $\rho_{\perp}$. Moreover, $G_{0}(\rho) \rightarrow 0$ as a bounded operator on this space as $|\rho| \rightarrow \infty$.

Proof. We fix some $\left(z^{0}, \rho_{\perp}^{0}\right)$, and show that $G_{0}(\rho)$ extends to be complex analytic in a neighborhood of this in $\mathbb{C}_{z} \times \mathbb{C}_{\rho_{\perp}}^{n-1}$. In fact, it is convenient to consider

$$
R_{0}(\rho)=e^{-i \rho_{\perp} \cdot w} G_{0}(\rho) e^{i \rho_{\perp} \cdot w} .
$$

Since the multipliers are holomorphic as maps

$$
e^{\gamma\langle w\rangle} H^{m}\left(\mathbb{R}^{n}\right) \rightarrow e^{\gamma^{\prime}\langle w\rangle} H^{m}\left(\mathbb{R}^{n}\right), \gamma<\gamma^{\prime},
$$

for $\left|\operatorname{Im} \rho_{\perp}\right|$ sufficiently small, and unitary for $\rho_{\perp}$ real, the original statement follows after we show that $R_{0}(\rho)$ extends analytically.

We do so by contour deformation on the Fourier transform side. Let $\xi=\left(\xi_{\|}, \xi_{\perp}\right)$ be the decomposition of $\xi$ according to the decomposition $\operatorname{span}(\{\nu\}) \oplus \operatorname{span}(\{\nu\})^{\perp}$ of $\mathbb{R}^{n}$. Thus, $\mathcal{F} R_{0}(\rho) \mathcal{F}^{-1}$ is a multiplication operator by $F^{-1}$ where

$$
F\left(\xi, z, \rho_{\perp}\right)=\left|\xi-\rho_{\perp}\right|^{2}+2\left(\xi-\rho_{\perp}\right) \cdot \rho=\xi_{\|}^{2}+2 z \xi_{\|}+\xi_{\perp}^{2}-\rho_{\perp}^{2} .
$$

Then

$$
\operatorname{Im} F=2 \operatorname{Im} z \xi_{\|}, \operatorname{Re} F=\xi_{\|}^{2}+2 \operatorname{Re} z \xi_{\|}+\xi_{\perp}^{2}-\rho_{\perp}^{2} .
$$

Thus the multiplication operator by $F^{-1}$ is singular where $F=0$, i.e. at

$$
\tilde{S}(\rho)=\left\{\xi: \xi_{\|}=0, \xi_{\perp}^{2}=\rho_{\perp}^{2}\right\},
$$


which is a sphere in the hyperplane $\xi_{\|}=0$.

It is convenient to break up $G_{0}(\rho)$ into two pieces by introducing a cutoff $\psi \in$ $\mathcal{C}_{c}^{\infty}\left(\mathbb{R}^{n}\right)$ that is identically 1 near $\tilde{S}\left(\rho^{0}\right)$. For instance, we may take

$$
\psi(\xi)=\phi\left(\xi_{\|}, \xi_{\perp}^{2}\right)
$$

with $\phi \in \mathcal{C}_{c}^{\infty}\left(\mathbb{R}^{2}\right)$, identically 1 near $\left(0,\left|\rho_{\perp}^{0}\right|^{2}\right)$. Then

$$
R_{0}(\rho)=R_{0}^{\prime}(\rho)+R_{0}^{\prime \prime}(\rho), R_{0}^{\prime}(\rho)=\mathcal{F}^{-1}\left(|\xi|^{2}+2 \xi \cdot \rho-\rho_{\perp}^{2}\right)^{-1} \psi(\xi) \mathcal{F} .
$$

Then $R_{0}^{\prime \prime}(\rho)$ is a Fourier multiplier by the function $(1-\psi(\xi)) F\left(\xi, z, \rho_{\perp}\right)^{-1}$, which is in fact a symbol of order -2 , analytic in $z$ and $\rho_{\perp}$ for $\operatorname{Im} \rho_{\perp}$ small, hence $R_{0}^{\prime \prime}(\rho)$ is analytic as a map $L^{2}\left(\mathbb{R}^{n}\right) \rightarrow H^{2}\left(\mathbb{R}^{n}\right)$.

To analyze $R_{0}^{\prime}(\rho)$, it is convenient to introduce polar coordinates in $\xi_{\perp}: \xi_{\perp}=r \omega$, $|\omega|=1, r \geq 0$. Then

$$
F=\xi_{\|}^{2}+2 z \xi_{\|}+r^{2}-\rho_{\perp}^{2} .
$$

Now, by Fubini's theorem $R_{0}(\rho) f=\mathcal{F}^{-1} F^{-1} \mathcal{F} f$ can be written as

$$
\begin{array}{r}
\left(R_{0}^{\prime}(\rho) f\right)(w)=(2 \pi)^{-n} \int_{\mathbb{R}} \int_{\mathbb{S}^{n-2}} \int_{0}^{\infty} e^{i r \omega \cdot w_{\perp}} e^{i \xi_{\|} w_{\|}}\left(\xi_{\|}^{2}+2 z \xi_{\|}+r^{2}-\rho_{\perp}^{2}\right)^{-1} \\
\psi\left(\xi_{\|}, r \omega\right)(\mathcal{F} f)\left(\xi_{\|}, r \omega\right) d r d \omega d \xi_{\|} .
\end{array}
$$

We divide the $\xi_{\|}$integral into two pieces, corresponding to $\xi_{\|} \geq 0$ and $\xi_{\|} \leq 0$. In each piece, we then deform the contour of the $r$ integral in a compact set disjoint from $\operatorname{supp}(1-\psi)$ near $r_{0}=\left|\rho_{\perp}^{0}\right|$ in such a way that $\operatorname{Im} r^{2}=2 \operatorname{Re} r \operatorname{Im} r$ and $\operatorname{Im} z \xi_{\|}$have the same sign on the contour. Note that the integrand is analytic in $r$ for $\operatorname{Im} r$ small and $\xi_{\|} \neq 0$.

Thus, suppose that $\operatorname{Im} z>0$. For $\xi_{\|}>0$, we deform the contour $[0,+\infty)_{r}$ near $r_{0}$ to a curve $\Gamma_{+}$so that $\operatorname{Im} r \geq 0$ on $\Gamma_{+}$and $r_{0}$ does not lie on the $\Gamma_{+}$. Now, $F$ never vanishes along $\Gamma_{+}$, provided that $\rho_{\perp}$ is close to $\rho_{\perp}^{0}$. Thus, extending $\psi$ to be 1 on $\Gamma_{+} \backslash[0,+\infty)$, and using that $\mathcal{F} f$ extends to be analytic in a tube $\left\{\xi \in \mathbb{C}^{n}:|\operatorname{Im} \xi|<\right.$ $\gamma\}$,

$$
\begin{gathered}
(2 \pi)^{-n} \int_{0}^{\infty} \int_{\mathbb{S}^{n-2}} \int_{0}^{\infty} e^{i r \omega \cdot w_{\perp}} e^{i \xi_{\|} w_{\|}}\left(\xi_{\|}^{2}+2 z \xi_{\|}+r^{2}-\rho_{\perp}^{2}\right)^{-1} \\
\psi\left(\xi_{\|}, r \omega\right)(\mathcal{F} f)\left(\xi_{\|}, r \omega\right) d r d \omega d \xi_{\|} \\
=(2 \pi)^{-n} \int_{0}^{\infty} \int_{\mathbb{S}^{n-2}} \int_{\Gamma_{+}} e^{i r \omega \cdot w_{\perp}} e^{i \xi_{\|} w_{\|}}\left(\xi_{\|}^{2}+2 z \xi_{\|}+r^{2}-\rho_{\perp}^{2}\right)^{-1} \\
\psi\left(\xi_{\|}, r \omega\right)(\mathcal{F} f)\left(\xi_{\|}, r \omega\right) d r d \omega d \xi_{\|},
\end{gathered}
$$

and on the right hand side we can allow $\rho_{\perp}$ to become complex, proving real analyticity of $R_{0}^{\prime}(\rho)$ in $\rho_{\perp}$, and extending it as an analytic family of operators $\mathcal{R}_{0}^{\prime}\left(z, \rho_{\perp}\right)$. This argument parallels the analytic continuation argument of [1, Chapter 1]. It is now easy to see that $\mathcal{R}_{0}^{\prime}\left(z, \rho_{\perp}\right)$ maps into $e^{\gamma\langle w\rangle} H^{2}\left(\mathbb{R}^{n}\right)$; indeed, it maps into $e^{\gamma\langle w\rangle} C_{\infty}^{\infty}\left(\mathbb{R}^{n}\right)$, where $C_{\infty}^{\infty}\left(\mathbb{R}^{n}\right)$ is the space of smooth functions which are bounded with all derivatives.

For $\xi_{\|}<0$ we proceed similarly, deforming the contour $[0,+\infty)_{r}$ near $r_{0}$ to a curve $\Gamma_{-}$so that $\operatorname{Im} r \geq 0$ on $\Gamma_{-}$and $r_{0}$ does not lie on the $\Gamma_{-}$. Again, we deduce real analyticity in $\rho_{\perp}$. 
The last part follows from the preceeding proposition since $e^{-\gamma|w|} L^{2} \subset L_{p}^{2} \subset L_{r}^{2} \subset$ $e^{\gamma|w|} L^{2}$.

Instead of the explicit contour deformation, we could have used the partial Fourier transform in $w_{\|}$, to deduce that

$$
G_{0}(\rho) f=e^{i \rho_{\perp} \cdot w} \mathcal{F}_{\|}^{-1}\left(\Delta_{\perp}+\xi_{\|}^{2}+2 z \xi_{\|}-\rho_{\perp}^{2}\right)^{-1} \mathcal{F}_{\|} e^{-i \rho_{\perp} \cdot w} f
$$

is real analytic in $\rho_{\perp}$ and analytic in $z$ by inserting step functions $1=H\left(\xi_{\|}\right)+H\left(-\xi_{\|}\right)$, and using the analyticity of

$$
\left(\Delta_{\perp}-\sigma\right)^{-1}: e^{-\gamma\left|w_{\perp}\right|} L^{2}\left(\operatorname{span}\{\nu\}^{\perp}\right) \rightarrow e^{\gamma\left|w_{\perp}\right|} L^{2}\left(\operatorname{span}\{\nu\}^{\perp}\right)
$$

in $\sigma$.

COROLlary 2.3. Suppose that $\gamma, \gamma_{0}>0$. Then the operator

$$
e^{-\gamma_{0}\langle w\rangle} \mathcal{G}_{0}\left(z, \rho_{\perp}\right) \in \mathcal{B}\left(e^{-\gamma|w|} L^{2}, e^{-\left(\gamma_{0}-\gamma\right)\langle w\rangle} H^{2}\right)
$$

is analytic in $z$ and in $\rho_{\perp}$ as a bounded operator between these spaces.

Corollary 2.4. Suppose that $V \in e^{-\gamma_{0}|w|} L^{\infty}$ and $\gamma_{0}>2 \gamma$, and let $U$ be as in Proposition 2.2. Then there exists a set

$$
\mathcal{E} \subset(\mathbb{C} \backslash \mathbb{R})_{z} \times U,
$$

which is given by the zeros of an analytic function and whose intersection with

$$
(\mathbb{C} \backslash \mathbb{R})_{z} \times\left(\mathbb{R}^{n-1} \backslash\{0\}\right)_{\rho_{\perp}}
$$

is bounded, such that $\left(\operatorname{Id}+V \mathcal{G}_{0}\left(z, \rho_{\perp}\right)\right)^{-1}$ exists in the complement of $\mathcal{E}$, and in a neighborhood of every point where it exists, $\left(\operatorname{Id}+V \mathcal{G}_{0}\left(z, \rho_{\perp}\right)\right)^{-1}$ is analytic with values in compact operators on $e^{-\gamma|w|} L^{2}$.

Proof. By the preceeding corollary, $e^{-\gamma_{0}\langle w\rangle} \mathcal{G}_{0}\left(z, \rho_{\perp}\right): e^{-\gamma|w|} L^{2} \rightarrow e^{-\left(\gamma_{0}-\gamma\right)\langle w\rangle} H^{2}$ is analytic in $z$ and $\rho_{\perp}$. But the inclusion $e^{-\left(\gamma_{0}-\gamma\right)\langle w\rangle} H^{2} \hookrightarrow e^{-\gamma|w|} L^{2}$ is compact, and $e^{\gamma_{0}\langle w\rangle} V \in L^{\infty}$, so $V \mathcal{G}_{0}\left(z, \rho_{\perp}\right)$ is an analytic family of compact operators on $e^{-\gamma|w|} L^{2}$. Moreover, as $|z| \rightarrow \infty$ or $\left|\rho_{\perp}\right| \rightarrow \infty, \rho_{\perp}$ real, $V \mathcal{G}_{0}\left(z, \rho_{\perp}\right)=V G_{0}(\rho) \rightarrow 0$ in norm. Thus, the conclusion follows by analytic Fredholm theory.

We write

$$
\mathcal{G}\left(z, \rho_{\perp}\right)=\mathcal{G}_{0}\left(z, \rho_{\perp}\right)\left(\operatorname{Id}+V \mathcal{G}_{0}\left(z, \rho_{\perp}\right)\right)^{-1}, G(\rho)=G_{0}(\rho)\left(\operatorname{Id}+V G_{0}(\rho)\right)^{-1} .
$$

We immediately deduce the following result.

Proposition 2.5. Suppose that $V \in e^{-\gamma_{0}|w|} L^{\infty}$,

$$
v_{z, \rho_{\perp}}=-\mathcal{G}\left(z, \rho_{\perp}\right) V \text {. }
$$

Then

$$
((\mathbb{C} \backslash \mathbb{R}) \times U) \backslash \mathcal{E} \ni\left(z, \rho_{\perp}\right) \mapsto v_{\rho}
$$

is an analytic function, with values in $e^{\gamma|w|} L^{2}$, for any $\gamma>0$. 
Corollary 2.6. Let $\nu \in \mathbb{S}^{n-1}$. Suppose that $V, V^{\prime} \in e^{-\gamma_{0}|w|} L^{\infty}$, and let $\mathcal{E}, \mathcal{E}^{\prime}$ be the exceptional sets of these two potentials. Then for $\left(z, \rho_{\perp}\right) \notin \mathcal{E},\left(z^{\prime}, \rho_{\perp}^{\prime}\right) \notin \mathcal{E}^{\prime}$ the pairing

$$
\int_{\mathbb{R}^{n}} u_{\rho}\left(V-V^{\prime}\right) u_{\rho^{\prime}}^{\prime}
$$

converges if $\left|\operatorname{Im} z+\operatorname{Im} z^{\prime}\right|<\gamma_{0}$, and is analytic in $z, z^{\prime}, \rho_{\perp}, \rho_{\perp}^{\prime}$.

Proof. We consider the strip $\left|\operatorname{Im} z+\operatorname{Im} z^{\prime}\right|<\gamma_{1}<\gamma_{0}, \gamma_{1}>0$. Let $\gamma \in\left(0,\left(\gamma_{0}-\right.\right.$ $\left.\left.\gamma_{1}\right) / 2\right)$. Then $1+v_{\rho}, 1+v_{\rho^{\prime}}^{\prime}$ are analytic in $\left(z, z^{\prime}, \rho_{\perp}, \rho_{\perp}^{\prime}\right)$ with values in $e^{\gamma|w|} L^{2}$. Hence,

$$
u_{\rho}\left(V-V^{\prime}\right) u_{\rho^{\prime}}^{\prime}=e^{i\left(\rho+\rho^{\prime}\right) \cdot w}\left(V-V^{\prime}\right)\left(1+v_{\rho}\right)\left(1+v_{\rho^{\prime}}^{\prime}\right)
$$

is analytic in $\left(z, z^{\prime}, \rho_{\perp}, \rho_{\perp}^{\prime}\right)$ with values in $L^{1}\left(\mathbb{R}^{n}\right)$. Integration preserves analyticity and proves the result.

3. Density of generalized eigenfunctions. In this section we relate tempered distributional eigenfunctions of $H=\Delta+V$ to its exponential eigenfunctions, constructed in the previous section.

We first introduce some notation. For $\lambda>0$, the free incoming Poisson operator is given by

$$
\tilde{P}_{+}(\lambda) g=c \int_{\mathbb{S}^{n-1}} e^{-i \sqrt{\lambda} w \cdot \omega} g d \omega_{a}, \quad g \in \mathcal{C}^{\infty}\left(\mathbb{S}^{n-1}\right), c=\lambda^{\frac{n-1}{4}} e^{-\frac{n-1}{4} \pi i}(2 \pi)^{-\frac{n-1}{2}} .
$$

The Poisson operator of $H$ is then

$$
P_{+}(\lambda) g=\tilde{P}_{+}(\lambda) g-R(\lambda+i 0)\left((H-\lambda) \tilde{P}_{+}(\lambda) g\right)=\tilde{P}_{+}(\lambda) g-R(\lambda+i 0) V \tilde{P}_{+}(\lambda) g .
$$

Note that for $g \in \mathcal{C}^{\infty}\left(\mathbb{S}^{n-1}\right), V \tilde{P}_{+}(\lambda) g$ is Schwartz, in fact decays exponentially, hence $R(\lambda+i 0)$ can be applied to it. For $g \in \mathcal{C}^{\infty}\left(\mathbb{S}^{n-1}\right)$,

$$
P_{+}(\lambda) g=e^{-i \sqrt{\lambda}|w|} g_{-}+e^{i \sqrt{\lambda}|w|} g_{+}+L^{2}\left(\mathbb{R}^{n}\right), g_{+}, g_{-} \in \mathcal{C}^{\infty}\left(\mathbb{S}^{n-1}\right), g_{-}=g .
$$

For such $g, P_{+}(\lambda) g$ is characterized by the property that it is the unique solution $u$ of $(H-\lambda) u=0$ which is of the form (3.1). The scattering matrix is then the operator

$$
S_{+}(\lambda): \mathcal{C}^{\infty}\left(\mathbb{S}^{n-1}\right) \rightarrow \mathcal{C}^{\infty}\left(\mathbb{S}^{n-1}\right), S_{+}(\lambda) g_{-}=g_{+} .
$$

There is also an incoming Poisson operator $P_{-}(\lambda)$ which is characterized by the fact that for $g \in \mathcal{C}^{\infty}\left(\mathbb{S}^{n-1}\right), P_{-}(\lambda) g$ is the unique solution $u$ of $(H-\lambda) u=0$ of the form

$$
P_{-}(\lambda) g=e^{-i \sqrt{\lambda}|w|} g_{-}+e^{i \sqrt{\lambda}|w|} g_{+}+L^{2}\left(\mathbb{R}^{n}\right), g_{+}, g_{-} \in \mathcal{C}^{\infty}\left(\mathbb{S}^{n-1}\right), g_{+}=g .
$$

In particular, for $g \in \mathcal{C}^{\infty}\left(\mathbb{S}^{n-1}\right)$,

$$
\overline{P_{-}(\lambda) g}=P_{+}(\lambda) \bar{g} .
$$

The S-matrix is related to the Poisson operator via the following boundary pairing. 
Proposition 3.1. [1, Lemma 2.2] Suppose that $\lambda>0$, and $V \in e^{-\gamma_{0}|w|} L^{\infty}$, $\gamma_{0}>0$. Suppose that $(H-\lambda) u_{+} \in L_{s}^{2},(H-\lambda) u_{-} \in L_{s}^{2}, s>1 / 2$, and

$$
\begin{aligned}
& u_{+}=e^{-i \sqrt{\lambda}|w|} g_{+-}+e^{i \sqrt{\lambda}|w|} g_{++}+L^{2}, \\
& u_{-}=e^{-i \sqrt{\lambda}|w|} g_{--}+e^{i \sqrt{\lambda}|w|} g_{-+}+L^{2},
\end{aligned}
$$

$g_{ \pm \pm} \in \mathcal{C}^{\infty}\left(\mathbb{S}^{n-1}\right)$. Then

$$
\left\langle u_{+},(H-\lambda) u_{-}\right\rangle-\left\langle(H-\lambda) u_{+}, u_{-}\right\rangle=2 i \sqrt{\lambda}\left(\left\langle g_{++}, g_{-+}\right\rangle-\left\langle g_{+-}, g_{--}\right\rangle\right) .
$$

REMARK 3.2. This is stated for $V \in \mathcal{C}_{c}^{\infty}\left(\mathbb{R}^{n}\right)$ in [1]. However, if $u_{+}, u_{-}$are as above, then $V u_{+} u_{-} \in e^{-\gamma|w|} L^{1}$ for $\gamma<\gamma_{0}$, hence the conclusion is equivalent to the corresponding statement with $H-\lambda$ replaced by $\Delta-\lambda$.

Let $R\left(\lambda^{\prime}\right)=\left(H-\lambda^{\prime}\right)^{-1}$ for $\lambda^{\prime} \in \mathbb{C} \backslash \mathbb{R}$. Let $f \in \mathcal{S}\left(\mathbb{R}^{n}\right), g \in \mathcal{C}^{\infty}\left(\mathbb{S}^{n-1}\right)$, and apply this proposition with

$$
u_{-}=R(\lambda-i 0) f=e^{-i \sqrt{\lambda}|w|} g_{--}+L^{2}, u_{+}=P_{+}(\lambda) g .
$$

We deduce that

$$
\left\langle u_{+}, f\right\rangle=-2 i \sqrt{\lambda}\left\langle g, g_{--}\right\rangle .
$$

Our density result is the following.

Proposition 3.3. Suppose that $V \in e^{-\gamma_{0}|w|} L^{\infty}$, and let $0<\gamma<\gamma^{\prime}<\gamma_{0}$. Then the set

$$
\mathcal{F}=\left\{P_{+}(\lambda) g_{+}: g_{+} \in \mathcal{C}^{\infty}\left(\mathbb{S}^{n-1}\right)\right\}
$$

is dense in the nullspace of $H-\lambda$ on $e^{\gamma|w|} L^{2}$ in the topology of $e^{\gamma^{\prime}|w|} L^{2}$.

Proof. Suppose that $f \in e^{-\gamma^{\prime}|w|} L^{2}$ is orthogonal to $\mathcal{F}$. Let $u_{-}=R(\lambda-i 0) f$. By (3.5), for all $g \in \mathcal{C}^{\infty}\left(\mathbb{S}^{n-1}\right),\left\langle g, g_{--}\right\rangle=0$ since $\left\langle f, P_{+}(\lambda) g\right\rangle$ vanishes by assumption. But $u_{-}=R_{0}(\lambda-i 0) f^{\prime}, f^{\prime}=f-V R(\lambda-i 0) f \in e^{-\gamma|w|} L^{2}$. Thus, $\mathcal{F} u_{-}$is the product of an analytic function, namely $\mathcal{F} f^{\prime}$, and $\left(|\xi|^{2}-(\lambda-i 0)\right)^{-1}$. Thus, $u_{-} \in L^{2}$ implies that $\mathcal{F} f^{\prime}$ vanishes on the sphere $|\xi|=\sqrt{\lambda}$. Hence $\mathcal{F} f^{\prime}=\left(\xi^{2}-\lambda\right) \phi$, with $\phi$ analytic in the strip $|\operatorname{Im} \xi|<\gamma^{\prime}$. Thus, $u_{-} \in e^{-\gamma|w|} L^{2}$ for $\gamma<\gamma^{\prime}$. Thus for $v \in e^{\gamma|w|} L^{2}$ with $(H-\lambda) v=0$,

$$
\langle f, v\rangle=\left\langle(H-\lambda) u_{-}, v\right\rangle=\left\langle u_{-},(H-\lambda) v\right\rangle=0,
$$

i.e. $f$ is orthogonal to the nullspace of $H-\lambda$ on $e^{\gamma|w|} L^{2}$. Thus, $\mathcal{F}$ is dense in this nullspace.

Our approach to the inverse problem relies on relating the S-matrices to the pairing (2.7). Thus, we consider two operators $H$ and $H^{\prime}$ induced by potentials $V$ and $V^{\prime}$ respectively, and show that the equality of the S-matrices at a fixed energy $\lambda$ implies the vanishing of an analogous pairing. For this we use the following consequence of Proposition 3.1 applied with $\Delta$ in place of $H$. Then

Proposition 3.4. Suppose that $\lambda>0$. Let $u_{+}=P_{+}(\lambda) g_{+}, u_{-}=P_{-}^{\prime}(\lambda) g_{-}$.

$$
\left\langle u_{+},(\Delta-\lambda) u_{-}\right\rangle-\left\langle(\Delta-\lambda) u_{+}, u_{-}\right\rangle=2 i \sqrt{\lambda}\left(\left\langle S_{+}(\lambda) g_{+}, g_{-}\right\rangle-\left\langle g_{+}, S_{-}^{\prime}(\lambda) g_{-}\right\rangle\right) .
$$


Corollary 3.5. Suppose $\lambda>0, S_{+}(\lambda)=S_{+}^{\prime}(\lambda)$. Let $u_{+}=P_{+}(\lambda) g_{+}, u_{-}=$ $P_{-}(\lambda) g_{-}$. Then

$$
\int_{\mathbb{R}^{n}}\left(V-V^{\prime}\right) u_{+} \overline{u_{-}}=0
$$

Similarly, if $u_{+}=P_{+}(\lambda) g_{+}, u_{-}=P_{+}(\lambda) g_{-}$, then.

$$
\int_{\mathbb{R}^{n}}\left(V-V^{\prime}\right) u_{+} u_{-}=0
$$

Proof. (3.7) follows from the preceeding proposition since $S_{-}^{\prime}(\lambda)^{*}=S_{+}^{\prime}(\lambda)$. Then (3.8) follows from (3.7) by applying the latter with $g_{-}$replaced by $\overline{g_{-}}$and using (3.3).

\section{$\square$}

4. Inverse results: Proof of Theorem 1.1. Let $\lambda>0$, and suppose that

$$
V, V^{\prime} \in e^{-\gamma_{0}|w|} L^{\infty}, \gamma_{0}>0 .
$$

Fix $\zeta \in \mathbb{R}^{n}$ such that $|\zeta|>2 \sqrt{\lambda}$, and let $\nu \in \mathbb{S}^{n-1}$ be orthogonal to $\zeta$, and let $\mu \in \mathbb{S}^{n-1}$ orthogonal to both $\zeta$ and $\nu$. For $t$ real, $t>\sqrt{\frac{1}{4}|\zeta|^{2}-\lambda}$, let

$$
\begin{aligned}
& \rho=\rho(t)=\frac{\zeta}{2}+\left(t^{2}-\frac{1}{4}|\zeta|^{2}+\lambda\right)^{1 / 2} \mu+i t \nu, \\
& \rho^{\prime}=\rho^{\prime}(t)=\frac{\zeta}{2}-\left(t^{2}-\frac{1}{4}|\zeta|^{2}+\lambda\right)^{1 / 2} \mu-i t \nu,
\end{aligned}
$$

so $\rho \cdot \rho=\lambda=\rho^{\prime} \cdot \rho^{\prime}$. By Corollary 2.6, the integral

$$
\int_{\mathbb{R}^{n}} u_{\rho}\left(V-V^{\prime}\right) u_{\rho^{\prime}}^{\prime}
$$

converges for all $t$, and is meromorphic in $t$ in a neighborhood of $\left(\sqrt{\frac{1}{4}|\zeta|^{2}-\lambda},+\infty\right)$. We use a density argument, Proposition 3.3, and Corollary 3.5 to show that this integral actually vanishes if $S_{+}(\lambda)=S_{+}^{\prime}(\lambda)$ and

$$
2 \sqrt{\lambda}<|\zeta|<\sqrt{4 \lambda+\gamma_{0}^{2}}
$$

Indeed, for $\sqrt{\frac{1}{4}|\zeta|^{2}-\lambda}<t<\gamma<\gamma_{0} / 2, u_{\rho^{\prime}}^{\prime}$ can be approximated by $P_{-}(\lambda) g_{-}$ in $e^{\gamma|w|} L^{2}$ due to Proposition 3.3. Similarly, $u_{\rho}$ can be approximated by $P_{+}(\lambda) g_{+}$ in $e^{\gamma|w|} L^{2}$. On the other hand, $V-V^{\prime}$ lies in $e^{-\gamma_{0}|w|} L^{2}$. Hence the product can be approximated in $L^{1}$ by a product which takes the form of the integrand of (3.8). The equality of the S-matrices implies that (3.8) vanishes, hence so does (4.2), i.e. we deduce the following result.

Proposition 4.1. Suppose that $\lambda>0, V, V^{\prime} \in e^{-\gamma_{0}|w|} L^{\infty}, S_{+}(\lambda)=S_{+}^{\prime}(\lambda)$. Then for $\zeta$ satisfying (4.3), $\rho, \rho^{\prime}$ given by (4.1) with $\left(z, \rho_{\perp}\right) \notin \mathcal{E},\left(z, \rho_{\perp}\right) \notin \mathcal{E}^{\prime}$,

$$
\int_{\mathbb{R}^{n}} u_{\rho}\left(V-V^{\prime}\right) u_{\rho^{\prime}}^{\prime}=0
$$

for $\sqrt{\frac{1}{4}|\zeta|^{2}-\lambda}<t<\gamma_{0} / 2$. 
The pairing in (4.4) is meromorphic in $t$ with $\operatorname{Re} t>\sqrt{\frac{1}{4}|\zeta|^{2}-\lambda}$ and $|\operatorname{Im} t|$ sufficiently small. It vanishes on an interval inside this domain by the proposition. Thus, (4.4) holds for all $t>\sqrt{\frac{1}{4}|\zeta|^{2}-\lambda}$. Then as $t \rightarrow \infty$, the integral on the left hand side of (4.4) converges to

$$
\int_{\mathbb{R}^{n}}\left(V-V^{\prime}\right) u_{\rho}^{0} u_{\rho^{\prime}}^{0}=\int_{\mathbb{R}^{n}}\left(V-V^{\prime}\right) e^{i \zeta \cdot w} d w
$$

since $v_{\rho} \rightarrow 0, v_{\rho}^{\prime} \rightarrow 0$ in $e^{\gamma|w|} L^{2}\left(\mathbb{R}^{n}\right)$ for any $\gamma>0$ and $V-V^{\prime} \in e^{-\gamma_{0}|w|} L^{\infty}\left(\mathbb{R}^{n}\right)$ with $\gamma_{0}>0$. But this is the Fourier transform of $V-V^{\prime}$, evaluated at $\zeta$. Hence the vanishing of (4.5) shows that the Fourier transform of $V-V^{\prime}$ vanishes on the shell (4.3). Since this Fourier transform is real analytic, as $V-V^{\prime} \in e^{-\gamma_{0}|w|} L^{\infty}$, we deduce that it vanishes everywhere, hence $V=V^{\prime}$. This completes the proof of Theorem 1.1.

\section{REFERENCES}

[1] R. B. Melrose, Geometric scattering theory, Cambridge University Press, 1995.

[2] R. G. Novikov and G. M. Khenkin, The $\bar{\partial}$-equation in the multidimensional inverse scattering problem, Uspekhi Mat. Nauk, 42(3(255))(1987), pp. 93-152.

[3] Roman G. Novikov, The inverse scattering problem at fixed energy for the three-dimensional Schrödinger equation with an exponentially decreasing potential, Comm. Math. Phys., 161(3)(1994), pp. 569-595.

[4] J. Sylvester and G. Uhlmann, A global uniqueness theorem for an inverse boundary value problem, Ann. of Math., 125 (1987), pp. 153-169.

[5] Gunther Uhlmann, Inverse boundary value problems and applications, Astérisque, 207(6)(1992), pp. 153-211. Méthodes semi-classiques, Vol. 1 (Nantes, 1991).

[6] RICARDO WEDER, Generalized limiting absorption method and multidimensional inverse scattering theory, Math. Methods Appl. Sci., 14(7)(1991), pp. 509-524. 
G. UHLMANN AND A. VASY 